\title{
In-flight measurement of the absolute energy scale of the Fermi Large Area Telescope
}

\begin{abstract}
M. Ackermann a , M. Ajello a , A. Allafort a , W.B. Atwood ${ }^{\text {b }}$, M. Axelsson ${ }^{\text {c,de, }}$, L. Baldini ${ }^{\text {f }}$, G. Barbiellini $^{\text {g,h }}$, D. Bastieri ${ }^{\mathrm{i} j \mathrm{j}}$, K. Bechtol ${ }^{\mathrm{a}}$, R. Bellazzini ${ }^{\mathrm{f}}$, B. Berenji ${ }^{\mathrm{a}}$, E.D. Bloom ${ }^{\mathrm{a}}$, E. Bonamente ${ }^{\mathrm{k}, 1}$, A.W. Borgland $^{\mathrm{a}}$, A. Bouvier $^{\text {b }}$, J. Bregeon ${ }^{\mathrm{f}}$, A. Brez ${ }^{\mathrm{f}}$, M. Brigida $^{\mathrm{m}, \mathrm{n}}$, P. Bruel $^{\mathrm{o}}$, R. Buehler ${ }^{\mathrm{a}}$, S. Buson $^{\mathrm{i}, \mathrm{j}}$, G.A. Caliandro ${ }^{\mathrm{p}}$, R.A. Cameron ${ }^{a}$, P.A. Caraveo ${ }^{\mathrm{q}}$, J.M. Casandjian ${ }^{\mathrm{r}}{ }^{\text {, C. Cecchi }}{ }^{\mathrm{k}, 1}$, E. Charles ${ }^{\mathrm{a}}$, A. Chekhtman ${ }^{\mathrm{s}, 1}$, J. Chiang ${ }^{\mathrm{a}}$, S. Ciprini ${ }^{\mathrm{t}, \mathrm{l}}$, R. Claus $^{\mathrm{a}}$, J. Cohen-Tanugi ${ }^{\mathrm{u}}$, S. Cutini ${ }^{\mathrm{v}}$, F. de Palma ${ }^{\mathrm{m}, \mathrm{n}}$, C.D. Dermer ${ }^{\mathrm{w}}$, S.W. Digel ${ }^{\mathrm{a}}$, E. do Couto e Silva ${ }^{a}$, P.S. Drell ${ }^{a}$, A. Drlica-Wagner ${ }^{a}$, R. Dubois ${ }^{a}$, T. Enoto ${ }^{a}$, L. Falletti $^{\mathrm{u}}$, C. Favuzzi ${ }^{\mathrm{m}, \mathrm{n}}$, S.J. Fegan ${ }^{\circ}$, W.B. Focke ${ }^{a}$, P. Fortin ${ }^{\circ}$, Y. Fukazawa ${ }^{\mathrm{x}}$, S. Funk ${ }^{\mathrm{a}}$, P. Fusco ${ }^{\mathrm{m}, \mathrm{n}}$, F. Gargano ${ }^{\mathrm{n}}$, N. Gehrels ${ }^{\mathrm{y}}$, S. Germani ${ }^{\mathrm{k}, \mathrm{l}}$, N. Giglietto ${ }^{\mathrm{m}, \mathrm{n}}$, F. Giordano ${ }^{\mathrm{m}, \mathrm{n}}$, M. Giroletti ${ }^{\mathrm{z}}$, T. Glanzman ${ }^{\mathrm{a}}$, G. Godfrey ${ }^{\mathrm{a}}$, I.A. Grenier ${ }^{\mathrm{r}}$, J.E. Grove $^{\mathrm{w}}$, S. Guiriec ${ }^{\text {aa }}$, D. Hadasch ${ }^{\mathrm{p}}$, M. Hayashida ${ }^{\mathrm{a}}$, E. Hays ${ }^{\mathrm{y}}$, R.E. Hughes ${ }^{\mathrm{ab}}$, G. Jóhannesson $^{\mathrm{ac}}$,

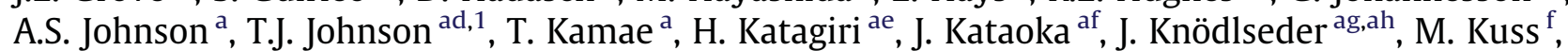
J. Lande $^{\mathrm{a}}$, L. Latronico ${ }^{\mathrm{f}}$, S.-H. Lee ${ }^{\mathrm{ai}}$, F. Longo ${ }^{\text {g,h }}$, F. Loparco ${ }^{\mathrm{m}, \mathrm{n}}$, M.N. Lovellette ${ }^{\mathrm{w}}$, P. Lubrano $^{\mathrm{k}, \mathrm{l}}$, G.M. Madejski ${ }^{\text {a }}$, M.N. Mazziotta ${ }^{n}$, J.E. McEnery ${ }^{\text {y,aj }}$, P.F. Michelson ${ }^{a}$, T. Mizuno ${ }^{\text {x }}$, A.A. Moiseev ${ }^{\text {ak,aj, }}$, C. Monte $^{\mathrm{m}, \mathrm{n}}$, M.E. Monzani ${ }^{\mathrm{a}}$, A. Morselli ${ }^{\text {al }}$, I.V. Moskalenko ${ }^{\mathrm{a}}$, S. Murgia ${ }^{\mathrm{a}}$, T. Nakamori ${ }^{\text {af }}$, M. Naumann-Godo ${ }^{\text {}}$, P.L. Nolan ${ }^{\text {a }}$, J.P. Norris ${ }^{\text {am }}$, E. Nuss ${ }^{\text {u }}$, T. Ohsugi ${ }^{\text {an }}$, A. Okumura ${ }^{\text {a,ao }}$, N. Omodei ${ }^{\text {a }}$, E. Orlando $^{\mathrm{a}, \mathrm{ap}}$, J.F. Ormes ${ }^{\mathrm{am}}$, M. Ozaki ${ }^{\mathrm{ao}}$, D. Paneque ${ }^{\mathrm{aq}, \mathrm{a}}$, J.H. Panetta ${ }^{a}$, D. Parent ${ }^{\mathrm{ar}, 1}$, M. Pesce-Rollins ${ }^{\mathrm{f}, *}$, M. Pierbattista ${ }^{\mathrm{r}}$, F. Piron ${ }^{\mathrm{u}}, \mathrm{S}$. Rainò ${ }^{\mathrm{m}, \mathrm{n}}$, R. Rando ${ }^{\mathrm{i}, \mathrm{j}}$, M. Razzano ${ }^{\mathrm{f}}$, A. Reimer $^{\mathrm{as}, \mathrm{a}}$, O. Reimer $^{\text {as,a }}{ }^{\text {, }}$ T. Reposeur ${ }^{\text {at }}$, S. Ritz ${ }^{\text {b }, ~ L . S . ~ R o c h e s t e r ~}{ }^{a}$, C. Sgrò ${ }^{f}$, E.J. Siskind ${ }^{\text {au }}$, P.D. Smith ${ }^{\text {ab }}$, G. Spandre ${ }^{f}$, P. Spinelli ${ }^{\mathrm{m}, \mathrm{n}}$, D.J. Suson ${ }^{\text {av }}$, H. Takahashi ${ }^{\text {an }}$, T. Tanaka ${ }^{\text {a }}$, J.G. Thayer ${ }^{\text {a }}$, J.B. Thayer ${ }^{\text {a }}$, D.J. Thompson ${ }^{\mathrm{y}}$, L. Tibaldo ${ }^{\mathrm{i}, \mathrm{j}, \mathrm{r}, 2}$, G. Tosti ${ }^{\text {k,l }}$, E. Troja ${ }^{\mathrm{y}, 3}$, T.L. Usher ${ }^{\mathrm{a}}$, J. Vandenbroucke ${ }^{\mathrm{a}}$, V. Vasileiou ${ }^{\mathrm{u}}$, G. Vianello $^{\mathrm{a}, \mathrm{aw}, \mathrm{c}}$, N. Vilchez $^{\text {ag,ah }}$, V. Vitale ${ }^{\text {al,ax }}$, A.P. Waite ${ }^{\text {a }}$, P. Wang ${ }^{\text {a }}$, B.L. Winer ${ }^{\text {ab }}$, K.S. Wood ${ }^{\text {w }}$, Z. Yang ${ }^{\text {ay,d }}$, S. Zimmer ${ }^{\text {ay,d }}$
\end{abstract}

${ }^{a}$ W.W. Hansen Experimental Physics Laboratory, Kavli Institute for Particle Astrophysics and Cosmology, Department of Physics, SLAC National Accelerator Laboratory, Stanford University, Stanford, CA 94305, USA

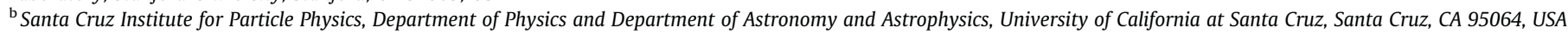

${ }^{\mathrm{c}}$ Department of Astronomy, Stockholm University, SE-106 91 Stockholm, Sweden

${ }^{\mathrm{d}}$ The Oskar Klein Centre for Cosmoparticle Physics, AlbaNova, SE-106 91 Stockholm, Sweden

e Department of Physics, Royal Institute of Technology (KTH), AlbaNova, SE-106 91 Stockholm, Sweden

${ }^{\mathrm{f}}$ Istituto Nazionale di Fisica Nucleare, Sezione di Pisa, I-56127 Pisa, Italy

${ }^{\mathrm{g}}$ Istituto Nazionale di Fisica Nucleare, Sezione di Trieste, I-34127 Trieste, Italy

${ }^{\mathrm{h}}$ Dipartimento di Fisica, Università di Trieste, I-34127 Trieste, Italy

${ }^{\mathrm{i}}$ Istituto Nazionale di Fisica Nucleare, Sezione di Padova, I-35131 Padova, Italy

${ }^{\mathrm{j}}$ Dipartimento di Fisica “G. Galilei”, Università di Padova, I-35131 Padova, Italy

${ }^{\mathrm{k}}$ Istituto Nazionale di Fisica Nucleare, Sezione di Perugia, I-06123 Perugia, Italy

${ }^{1}$ Dipartimento di Fisica, Università degli Studi di Perugia, I-06123 Perugia, Italy

m Dipartimento di Fisica "M. Merlin" dell'Università e del Politecnico di Bari, I-70126 Bari, Italy

${ }^{\mathrm{n}}$ Istituto Nazionale di Fisica Nucleare, Sezione di Bari, 70126 Bari, Italy

${ }^{\circ}$ Laboratoire Leprince-Ringuet, École polytechnique, CNRS/IN2P3, Palaiseau, France

${ }^{\mathrm{p}}$ Institut de Ciències de l'Espai (IEEE-CSIC), Campus UAB, 08193 Barcelona, Spain

${ }^{\mathrm{q}}$ INAF-Istituto di Astrofisica Spaziale e Fisica Cosmica, I-20133 Milano, Italy

${ }^{\mathrm{r}}$ Laboratoire AIM, CEA-IRFU/CNRS/Université Paris Diderot, Service d'Astrophysique, CEA Saclay, 91191 Gif sur Yvette, France

${ }^{\mathrm{s}}$ Artep Inc., 2922 Excelsior Springs Court, Ellicott City, MD 21042, USA

${ }^{\mathrm{t}}$ ASI Science Data Center, I-00044 Frascati (Roma), Italy

uLaboratoire Univers et Particules de Montpellier, Université Montpellier 2, CNRS/IN2P3, Montpellier, France

${ }^{\mathrm{v}}$ Agenzia Spaziale Italiana (ASI) Science Data Center, I-00044 Frascati (Roma), Italy

${ }^{\mathrm{w}}$ Space Science Division, Naval Research Laboratory, Washington, DC 20375-5352, USA

${ }^{\mathrm{x}}$ Department of Physical Sciences, Hiroshima University, Higashi-Hiroshima, Hiroshima 739-8526, Japan

${ }^{\mathrm{y}}$ NASA Goddard Space Flight Center, Greenbelt, MD 20771, USA

${ }^{\mathrm{z}}$ INAF Istituto di Radioastronomia, 40129 Bologna, Italy

aa Center for Space Plasma and Aeronomic Research (CSPAR), University of Alabama in Huntsville, Huntsville, AL 35899, USA

${ }^{\mathrm{ab}}$ Department of Physics, Center for Cosmology and Astro-Particle Physics, The Ohio State University, Columbus, OH 43210, USA

${ }^{\mathrm{ac}}$ Science Institute, University of Iceland, IS-107 Reykjavik, Iceland 


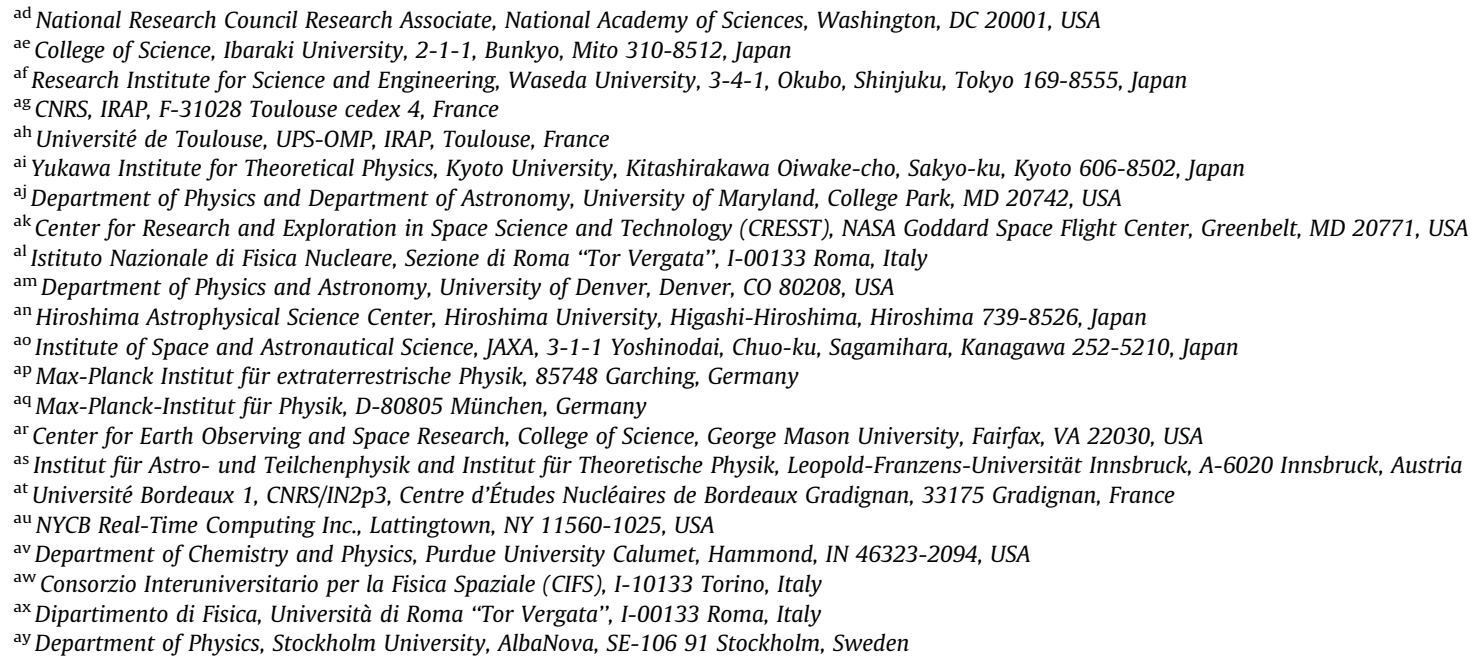

\section{A R T I C L E I N F O}

\section{Article history:}

Received 20 June 2011

Received in revised form 18 October 2011

Accepted 21 October 2011

Available online 30 October 2011

\section{Keywords:}

Cosmic-rays

Geomagnetic cutoff

Absolute energy scale

Fermi Large Area Telescope

\begin{abstract}
A B S T R A C T
The Large Area Telescope (LAT) on-board the Fermi Gamma-ray Space Telescope is a pair-conversion telescope designed to survey the gamma-ray sky from $20 \mathrm{MeV}$ to several hundreds of $\mathrm{GeV}$. In this energy band there are no astronomical sources with sufficiently well known and sharp spectral features to allow an absolute calibration of the LAT energy scale. However, the geomagnetic cutoff in the cosmic ray electron-plus-positron (CRE) spectrum in low Earth orbit does provide such a spectral feature. The energy and spectral shape of this cutoff can be calculated with the aid of a numerical code tracing charged particles in the Earth's magnetic field. By comparing the cutoff value with that measured by the LAT in different geomagnetic positions, we have obtained several calibration points between $\sim 6$ and $\sim 13 \mathrm{GeV}$ with an estimated uncertainty of $\sim 2 \%$. An energy calibration with such high accuracy reduces the systematic uncertainty in LAT measurements of, for example, the spectral cutoff in the emission from gamma ray pulsars.
\end{abstract}

(C) 2011 Elsevier B.V. All rights reserved.

\section{Introduction}

The Large Area Telescope (LAT) is the primary instrument on the Fermi Gamma-ray Space Telescope, which was launched on June 11,2008 . The LAT is a $4 \times 4$ modular array of identical towers, each comprised of a tracker/converter (TKR) and a calorimeter (CAL), surrounded by an anti-coincidence detector (ACD). Each TKR module contains 18 layers of tungsten foil and paired silicon strip detector $x-y$ tracking planes. Each CAL module contains a hodoscopic array of $96 \mathrm{CSI}$ ( $\mathrm{Tl}$ ) scintillating crystals 8.6 radiation lengths deep. High energy gamma rays predominantly convert in the TKR into electron-positron pairs, and the energy of the developing electromagnetic shower is measured in the CAL. To cover the energy range of the instrument the individual CAL crystals must be able to accurately measure energy deposits between $2 \mathrm{MeV}$ and 60 $\mathrm{GeV}$. To accomplish this, each CAL crystal can measure the deposited energy in four different overlapping energy ranges, namely 2-100 MeV, $2 \mathrm{MeV}-1 \mathrm{GeV}, 30 \mathrm{MeV}-7 \mathrm{GeV}$, and $30 \mathrm{MeV}-70 \mathrm{GeV}$.

The process of converting the scintillation light measured in each individual CAL crystal to an energy of the incident photon can be roughly divided into two phases. The first phase is the calculation of the energy deposition in each crystal (hereafter, the

\footnotetext{
* Corresponding author.

E-mail address: melissa.pesce.rollins@pi.infn.it (M. Pesce-Rollins).

${ }^{1}$ Resident at Naval Research Laboratory, Washington, DC 20375, USA.

2 Partially supported by the International Doctorate on Astroparticle Physics (IDAPP) program.

3 NASA Postdoctoral Program Fellow, USA.
}

"crystal energy"), which relies on calibration constants derived from the measured signals from sea-level muons and on-orbit cosmic ray protons together with constants derived from an on-orbit electronic charge-injection system. The second phase is event reconstruction, which is comprised of a set of algorithms that account for energy deposited in the TKR, leakage of the shower out the back and sides of the CAL, and energy lost in gaps between the towers. We developed the energy reconstruction algorithms [1] from a detailed instrument model and Monte Carlo simulation based on GEANT4.

To verify the fidelity of the instrument model and Monte Carlo simulation, we performed an extensive beam test campaign in 2006 at CERN and the GSI heavy ion accelerator laboratory. To eliminate the handling and shipping risks to the flight detector subsystems, and to reduce cost and schedule impacts, these tests were not done on the full LAT but on a Calibration Unit (CU), which consisted of two fully-populated flight-spare TKR modules and three fully-populated flight-spare CAL modules. A more detailed description of the beam test as well as the energy reconstruction algorithms will be presented in Section 2 .

To perform an in-flight verification of the absolute energy scale of the LAT it is necessary to find an astrophysical source with a spectral feature whose absolute energy peak and shape are well known. Because sources in the LAT energy band exhibit smooth spectral forms, it is not trivial to satisfy this requirement. Furthermore, the LAT is far more sensitive than any previous gamma-ray telescope, so it is not possible to use existing measurements of celestial sources to perform this calibration. The LAT has shown that gamma-ray pulsars typically exhibit spectral cutoffs in the 
1-10 GeV range [2] and, for bright pulsars, the spectral cutoff energy can be measured with statistical uncertainty approaching $1 \%$ [3]. If the systematic uncertainty on the energy scale in this energy range can be reduced to the few-percent level, precise measurements of spectral cutoff can address detailed questions of the emission physics and geometry in pulsars [3]. In the orbit of the Fermi observatory (inclination of $25.6^{\circ}$ and altitude of $565 \mathrm{~km}$ ), the geomagnetic cutoff in the cosmic-ray electron plus positron (CRE) spectrum can serve as such a source in the range between $\sim 6$ and $\sim 13 \mathrm{GeV}$. Electrons and positrons are a good calibration source because they interact in the LAT CAL in the same way that photons do: they both produce electromagnetic showers. The energy measurement bias coming from the slight differences between CRE and photons interactions in the LAT is much less than $1 \%$ [4]. The capabilities of the LAT to measure the CRE spectrum have been well demonstrated $[4,5]$. The cutoff rigidity ${ }^{4}$ can be predicted by numerically tracing particle trajectories in the Earth's magnetic field, and the comparison of the predicted and measured values provides the opportunity to perform this validation.

In order to measure the deviation between the reconstructed electron spectrum and the calculated value as a function of energy, this analysis is performed in several Mcllwain $L$ intervals. The McIlwain $L$-parameter [6] is a parameter describing a set of the Earth's magnetic field lines, in particular those which cross the Earth's magnetic equator at a number of Earth-radii equal to the $L$-value. Magnetically equivalent positions (from the standpoint of the incoming charged particle) around the world will by definition have the same McIlwain $L$ values, therefore making this parameter particularly convenient for characterizing cutoff rigidities [7]. The orbital inclination of the Fermi orbit fixes the range of Mcllwain $L$ values accessible for this analysis to $1.00-1.72$.

\section{LAT energy calibration}

The absolute energy scale of the LAT is defined by comparing the signals in the CAL crystals with the amount of energy a Monte Carlo simulation indicates should be deposited by on-orbit relativistic protons. The details of the energy calibration, referred to as the "proton inter-range calibration", are given in [8]. To perform this calibration, we first correct the observed CAL signals for electronic non-linearities (as measured by an electronic charge-injection process) and for position-dependent scintillation response (as measured by a direct calibration with sea-level muons and on-orbit with protons). We then compare the corrected CAL signals with the distribution of deposited energies predicted by a GEANT4 simulation of the on-orbit spectrum of primary cosmic-ray protons passing through the LAT. This calibrates the highest-gain, lowestenergy range of the CAL readout. The remaining gain ranges [1] of the CAL readout are calibrated by enforcing that adjacent gain ranges give the same measured energy in the regions of energy space in which they overlap [8].

The Galactic cosmic-ray (GCR) element abundance peaks could in principle be used to calibrate the higher energy ranges of the LAT; however, the scintillation efficiency of heavy ions in CsI ( Tl) is not identical to that of electromagnetic showers by an element-dependent factor that is not well known or measured. We measured the relative scintillation efficiencies of sub-relativistic ions in CSI ( $\mathrm{Tl})$ crystals in a series of beam tests [9], but we do not have sufficient confidence that these efficiencies apply at relativistic energies. Thus, we are unable to relate the observed signals

\footnotetext{
${ }^{4}$ Rigidity is defined as the particle momentum divided by its charge. The cutoff we are measuring in the CRE spectrum is not the vertical cutoff but rather averaged over all angles.
}

from GCR element peaks to electromagnetic shower energy depositions accurately enough to calibrate the LAT energy scale.

The geomagnetic cutoff in the CRE spectrum (measured in the energy range $\sim 6$ to $\sim 13 \mathrm{GeV}$ ) is a good alternative source to calibrate the higher energy ranges of the CAL readout. At these energies, the maximum energy per crystal is of the order of $\sim 1 \mathrm{GeV}$, and will be read out in the higher energy ranges of the CAL. As a reference, $1 \mathrm{GeV}$ of energy per crystal is equivalent to 100 times what a minimum ionizing particle releases per crystal.

The LAT CU was also calibrated using sea-level cosmic-ray muons, but the absolute energy scale was then cross-checked against calibrated beam lines at CERN, showing an energy deposit systematically larger than expected. Such a direct calibration relies on the knowledge of the incoming beam energy (determined with a $1 \%$ accuracy), the geometry of the beam line and the CU detector, the Monte Carlo of the electromagnetic shower development within the detector, as well as on a good control of the environmental effects that might influence the CU response (temperature, humidity, exceedingly high particle rates).

The energy reconstruction of the LAT (as well as of the CU) is based on three different algorithms: a parametric correction based on the barycenter of the shower, a fit to the shower profile taking into account the longitudinal and transverse development of the shower, and a maximum likelihood fit based on the correlation between the total deposited energy, the energy deposited in the last layer of the CAL and the number of tracker hits. For each event, the best energy reconstruction method is selected by means of a classification tree analysis described in [1]. We would like to emphasize that at the energies considered in this analysis (i.e. between $\sim 6$ and $\sim 13 \mathrm{GeV}$ ) the correction factors for losses due to leakage out of the detector are reasonably small (on average of the order of $30 \%$ ) when compared to higher energies (of the order of $50 \%$ for 100 's of $\mathrm{GeV}$ ) and therefore the method described here is testing a mixture of the crystal energy calibration and the leakage corrections.

Following the beam test campaign, the GEANT4 simulation of the $\mathrm{CU}$ was updated to reflect the best available description of the $\mathrm{CU}$ detector, the beam line, and the particle interactions. Most notably, the routines describing the Landau-Pomeranchuk-Migdal effect (LPM [10]) in electromagnetic shower developments were updated after finding that initial GEANT4 implementations were not providing a satisfactory description of the shower longitudinal development. The energy resolution for all three available energy reconstruction methods was measured to be consistent with expectations. However despite these improvements, the reconstructed energy in the CU was consistently higher than the beam energy by $\sim 9 \%$, on average, with further fluctuations of $\sim 5 \%$, depending on incoming beam energy (5-282 GeV), angle (0-60 degrees) and position of the crystal within the shower [4,11].

The energy scale factor derived from the direct $\mathrm{CU}$ calibration was not applied to the LAT because of the differences in the beam test and on-orbit environment, particularly the distribution and rate of the particles incident on the calorimeter, and the temperature variations for the $\mathrm{CU}$ during the data taking, which were considered responsible for the different LAT and CU energy scales.

\section{Particle tracing}

Geomagnetic cutoff rigidities can be obtained by tracing cosmic-ray trajectories in a model of the Earth's magnetic field. The standard mathematical description of the Earth's magnetic field is given by the International Geomagnetic Reference Field models (IGRF) [12]. These models consist of the Gauss coefficients defining the spherical harmonic expansion of the geomagnetic potential up to a given order. In the IGRF-11 model, used for this analysis, the 


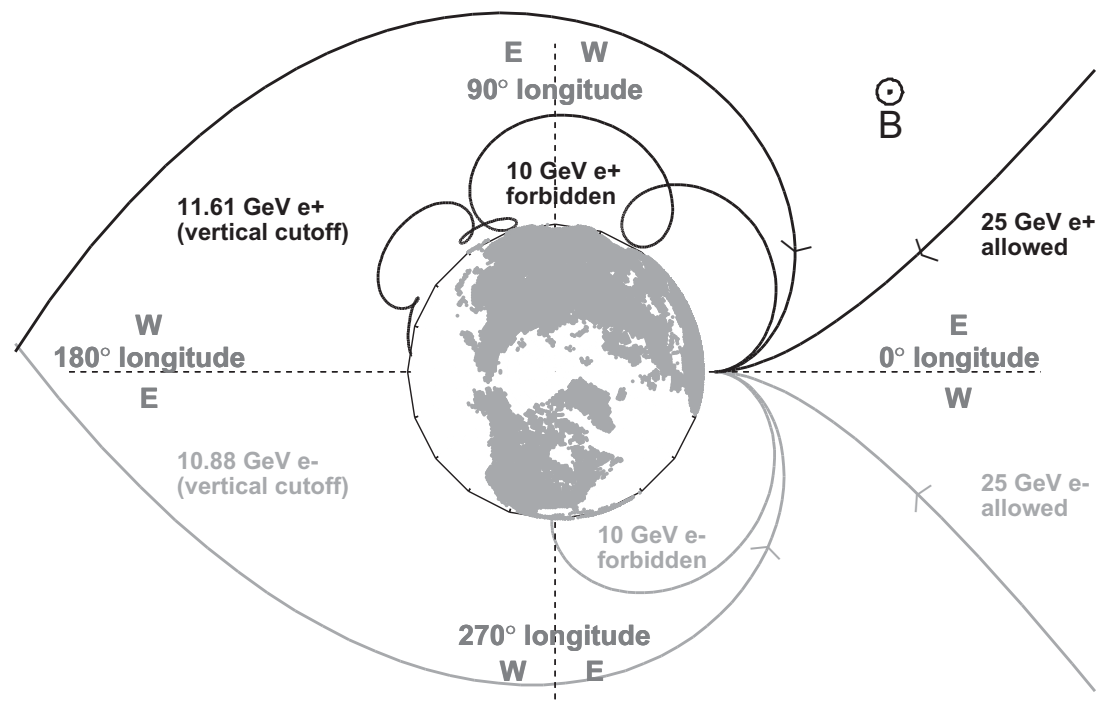

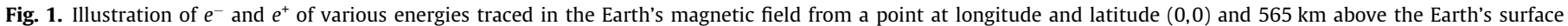

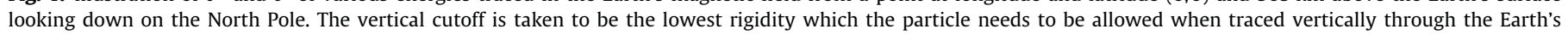
magnetic field. As the rigidity of the particle decreases the amount of geomagnetic bending increases and when the trajectory intersects the Earth it is taken to be forbidden

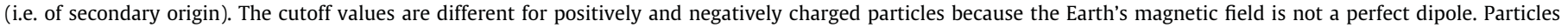
labeled as allowed are taken to be of Galactic origin. Earth and trajectories are to scale.

maximum multipole moment of the expansion is the 13 th. The IGRF model is computed by the participating members of the international association of geomagnetism and aeronomy (IAGA) working group V-MOD [12] which is supported by the organizations involved in operating magnetic survey satellites, observatories, magnetic survey programs and World Data Centers. IGRF-11 provides a definitive main field model for epoch 2005, a main field model for 2010, and a linear predictive secular variation model for 2010-2015. We use the particle trajectory tracing code (hereafter tracer) developed by Smart and Shea [7] and the IGRF-11 in this analysis to measure the geomagnetic cutoff.

Since it is difficult to trace the trajectory of an incoming particle through the magnetic field and expect it to intersect the precise location desired, it is more efficient to calculate the trajectory in the reverse direction (i.e. trace out a positron to mimic an incoming electron). Therefore the starting point of the trajectory is given by the geographic coordinates, altitude and orientation of the spacecraft and the trajectories are propagated using IGRF-11 [12].

At each of a grid of locations covered by the Fermi orbit, evaluated every $15 \mathrm{~s}$ from Aug 2008 through Jul 2009, we simulated an ensemble of test particles. We selected energies of the test particles according to the power-law spectrum measured by the LAT [4], and generated both electrons and positrons in the abundance ratio measured by PAMELA [13]. We use the tracer code to determine for each test particle whether it could have originated from outside the geomagnetosphere. Trajectories that eventually intersect the Earth's atmosphere ${ }^{5}$ are rejected and labeled as forbidden (secondaries hereafter). Trajectories that reach 20 Earth radii are accepted as allowed (or of Galactic origin, primaries hereafter). Fig. 1 illustrates a few sample trajectories, both forbidden and allowed.

We selected electron and positron data from the first year of Fermi LAT data taking (Aug 2008 through Jul 2009) using the methods described in [4] for the $100 \mathrm{MeV}$ to $100 \mathrm{GeV}$ energy range. In Fig. 2 are the distributions (for $E>30 \mathrm{GeV}$ ) of the reconstructed angle with respect to local zenith (upper panel) and azimuth (lower panel) for the flight data and for the tracer output. The overall

\footnotetext{
${ }^{5}$ Distance taken to be $20 \mathrm{~km}$ from the Earth's surface.
}
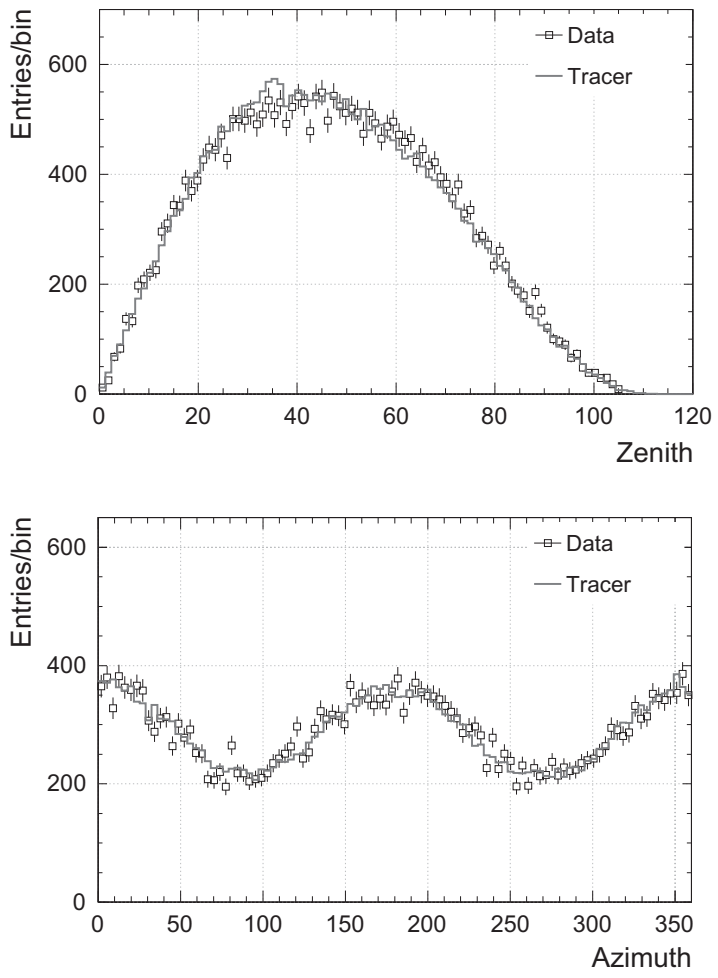

Fig. 2. The reconstructed angle with respect to local zenith (upper panel) and azimuth (lower panel) for data and tracer. In the lower panel, North is at $0^{\circ}$, East at $90^{\circ}$, South at $180^{\circ}$ and West at $270^{\circ}$. The LAT acceptance for electrons and positrons as well as the rocking profile have been convolved in these distributions and influence their shape. Both are averaged over the orbit and for energies greater than $30 \mathrm{GeV}$ in order to compare the distribution of primary CREs. There is an overall good agreement between data and tracer.

agreement between flight data and the tracer output is very good, demonstrating that the angular distributions have been well described. 


\section{Analysis}

\subsection{Estimating the fraction of primary cosmic ray electrons}

The population of CREs in low Earth orbit is a mixture of primary and secondary cosmic rays, where primary implies of extraterrestrial origin and secondary implies a mixture of splash and reentrant particles produced in the interaction of primaries in the Earth's atmosphere. The trajectories of the secondary particles are extremely difficult to simulate reliably. By definition our tracing code only provides information on the allowed (i.e. primary) particles. As a consequence it is necessary to estimate the fraction of secondary particles, as a function of energy, from the flight data. This constitutes one of the most delicate aspects of the analysis because the cutoff rigidity is found by fitting the spectrum (as will be described in detail in Section 4.2) and its shape is influenced by the fraction of secondaries.

The azimuthal distribution in Earth centered coordinates of the secondary population is different from that of the primary particles. We can exploit this fact and perform a template fit to identify the fraction of each population. To perform this task it is first necessary to choose the appropriate templates to describe the populations. It is safe to assume that the population at low energy in flight data ( $E \ll E_{c}$, where $E_{c}$ is the energy corresponding to the cutoff rigidity) is predominately composed of secondary particles, and can therefore be used as a template. The output from the tracer code provides the template for the primaries. Fig. 3 shows an example of the template fitting performed to estimate the fraction of primaries in the interval $1.0<$ Mcllwain $L<1.14$. The gradual transition from a pure sample of secondary particles (top left panel, for energies between 2 and $4 \mathrm{GeV}$ ) to that of only primaries (bottom right panel, for energies between 16 and $18 \mathrm{GeV}$ ) is evident.
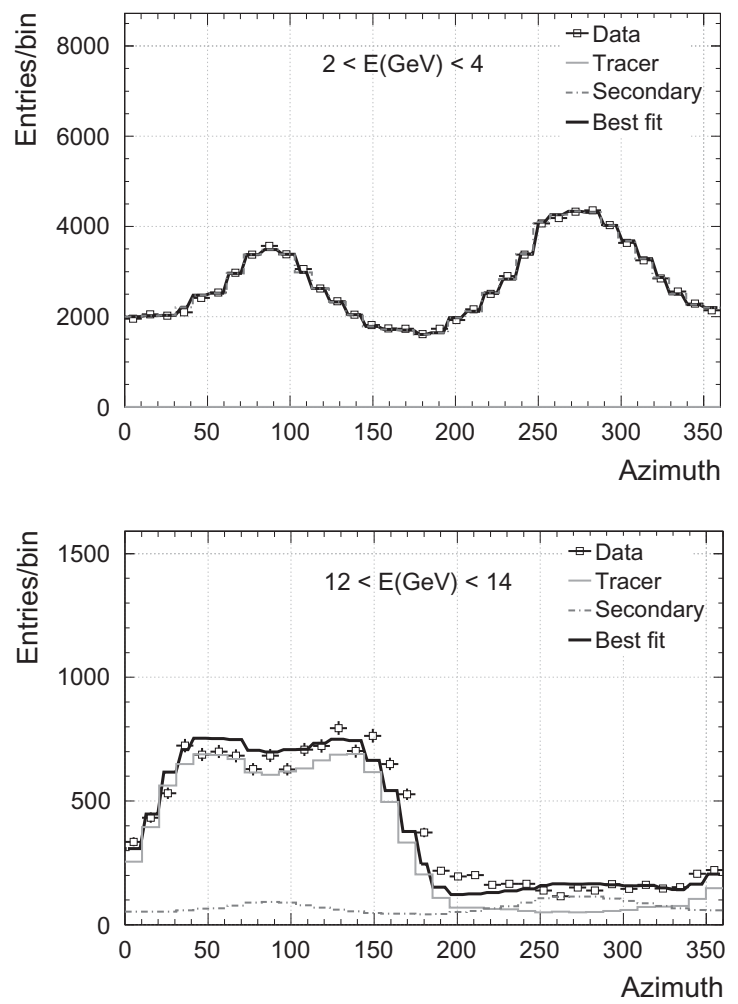

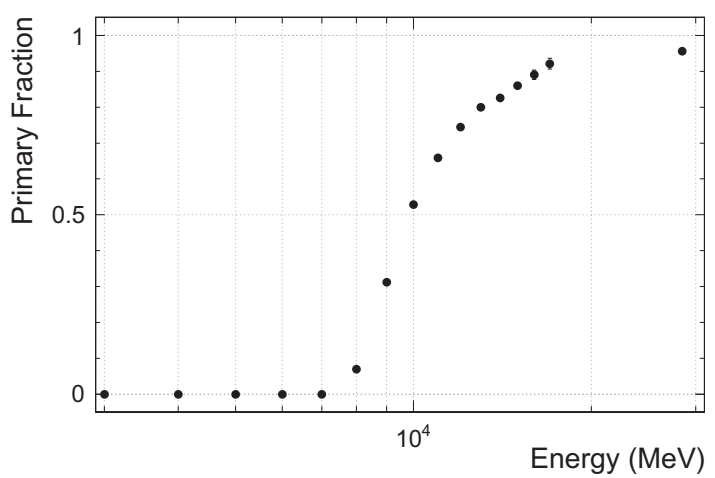

Fig. 4. Fraction of primary electrons and positrons as a function of energy for the Mcllwain $L$ interval $1.00<L<1.14$. Note that the primary fraction does not reach $100 \%$ because contamination from hadrons has not yet been accounted for. This is done when reconstructing the primary spectrum as described in Section 4.2.

For reference, the cutoff energy in this McIlwain $L$ interval is $\sim 13 \mathrm{GeV}$. For simplicity, only four energy intervals are shown. However the analysis has been performed in 16 overlapping energy intervals. The resulting value for the fraction of primaries as a function of energy for this same McIlwain $L$ interval is shown in Fig. 4.

\subsection{Measuring the cutoff rigidity}

The primary $e^{-}+e^{+}$spectrum can be parameterized by [4]:

$$
\frac{d N(E)}{d E}=\frac{c E^{-\Gamma}}{\left(1+\left(E / E_{c}\right)^{-6}\right)}
$$
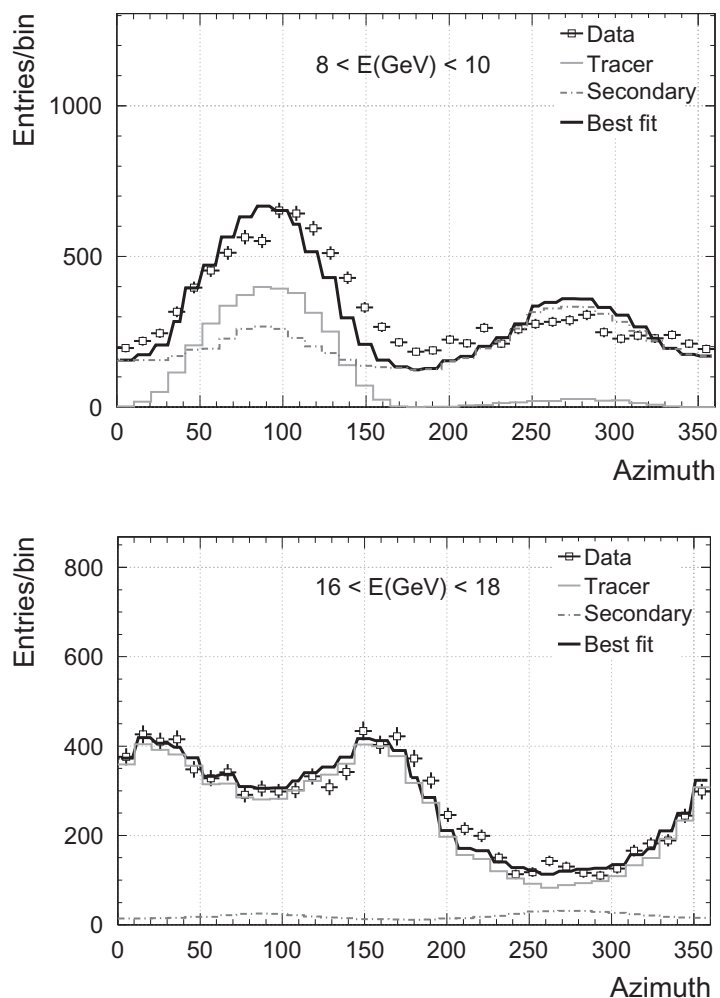

Fig. 3. Azimuthal distribution for flight data, tracer and secondaries in the McIlwain $L$ interval $1.0<L<1.14$ averaged over all zenith angles. The black distribution is the linear

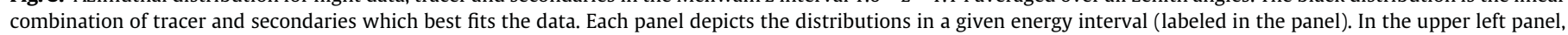
where the secondary population is dominant, a maximum is evident in the westward direction where the secondary positrons are dominant. With increasing energy, the

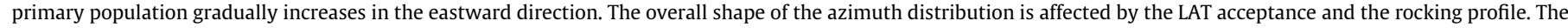
cutoff rigidity in this Mcllwain $L$ range is $\sim 13 \mathrm{GeV}$. The fraction of primaries evaluated from this template fitting is shown in Fig. 4 . 
where $\Gamma$ is the spectral index and $E_{c}$ is the energy corresponding to the cutoff rigidity. We fit the primary CRE spectrum (both data and tracer) with Eq. (1) to get the value of the cutoff rigidity.

To reconstruct the CRE primary spectrum it is first necessary to remove the secondary population from the count spectrum and then correct for the hadronic contamination. The residual contamination from hadrons, $h$, is estimated by applying the CRE event selection to the on-orbit simulation used by the Fermi LAT collaboration. We found that this residual hadron contamination ranges between $\sim 15 \%$ below the cutoff and $\sim 5 \%$ above with an estimated uncertainty on the absolute hadron flux of $20 \%$. The on-orbit simulation is based on data from CR experiments and includes all the components of the Galactic cosmic rays as well as the re-entrant and splash Earth albedo particles. A detailed description of this simulation can be found in [4]. The residual contamination due to secondaries, $s$, is estimated as described in Section 4.1. The count spectrum is multiplied by the purity factor defined as $P \cdot F=(1-h) \cdot(1-s)$ in each energy bin. This background-subtracted count spectrum is divided by the effective geometry factor (EGF) [4] and the width of the energy interval, to obtain the final spectrum.

The tracer count spectra are reconstructed by requiring the allowed condition and dividing by the width of the energy interval. To properly compare the cutoff rigidities, the tracer spectra have been binned in measured energy (i.e., they have been convolved using the energy resolution of the Fermi LAT for electrons and positrons [4]). No efficiency correction is required for the tracer spectra. To measure the spectra in Mcllwain $L$ intervals an extra cut specifying the interval is applied to both data and tracer.

A comparison between the counts spectra measured from flight data and from the tracer output is shown in Fig. 5. The shapes of the spectra are in good agreement, indicated by the ratio of the two depicted in the lower panel of the figure. Both the reconstruction and fitting procedures are applied to each Mcllwain $L$ interval considered in this analysis.

\subsection{Assessment of the systematic uncertainties}

The main sources of systematic uncertainty in this analysis are: the choice of the energy interval in which to fit the spectrum, the choice of the energy interval for the secondary template, the
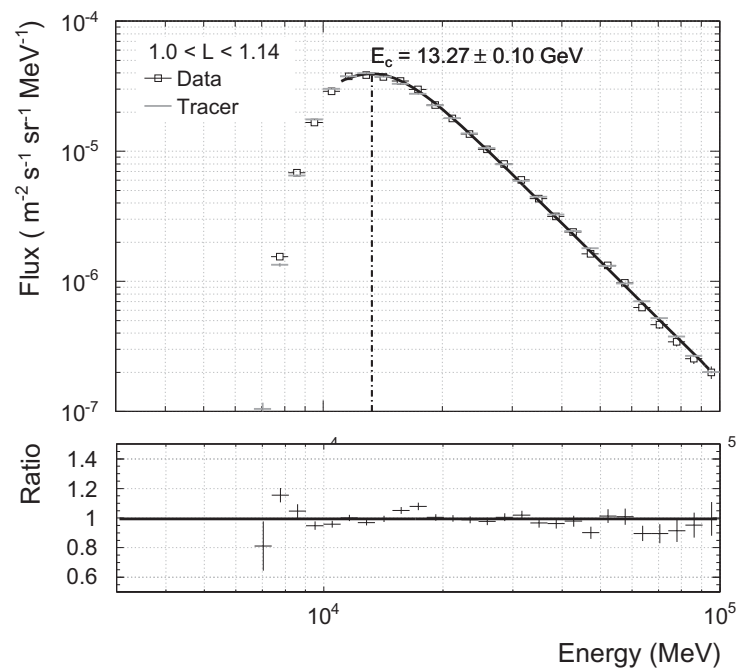

Fig. 5. Energy spectrum of data and tracer for the Mcllwain $L$ interval $1.0<L<1.14$. The black line is a fit to the data using Eq. (1) and the resulting cutoff energy has a value of $13.27 \pm 0.10 \mathrm{GeV}$, and is indicated by the dot dashed line in the figure. The lower panel depicts the ratio of the two spectra. accuracy of the geomagnetic field model, and the accuracy of the Monte Carlo simulations used for the estimation of the residual hadron contamination as well as the evaluation of the EGF.

To estimate the systematic uncertainty from the choice of energy interval used for the spectral fits, we performed a series of fits over a range of energy intervals narrower and broader than the chosen interval and calculated the root-mean square (rms) of the distribution of resulting values of the cutoff energy. The rms was found to be no greater than $0.8 \%$ for all the Mcllwain $L$ intervals.

Since the key ingredients in the calculation of the fraction of CRE primaries are the secondary and primary templates, it is crucial to validate them. The tracer output (used as the template for the primaries) can be validated directly with the Fermi LAT flight data, as was described in Section 3. The secondary template is more difficult to validate given that the output from the tracer code does not provide any information regarding the secondary populations. As stated in Section 4.1, it is safe to assume that the population at low energy in flight data $\left(E \ll E_{c}\right)$ is predominantly composed of secondary particles. However the choice of the energy interval is not well determined, and can therefore be a source of uncertainty. This choice can in fact influence the shape of the spectrum and thus the final value for the cutoff rigidity. To investigate its effect, we chose several different energies ranges in which to define our secondary templates (for each McIlwain $L$ interval) by varying both the width of the energy interval as well as the distance from the cutoff energy. With the resulting fraction of primaries obtained from each template we reconstructed the spectrum and obtained the cutoff rigidity following the procedure described in Section 4.2. The spread of the ratio of the cutoffs (found to be no greater than $\sim 2 \%$ for all Mcllwain $L$ intervals) gives an estimate for the uncertainty due to the secondary template.

The accuracy of the cutoffs derived from the particle tracing code is limited by the uncertainty of the geomagnetic field model. Several cross checks on the accuracy of the predicted rigidity cutoffs have been performed using satellite experiments, in particular the cosmic-ray isotope experiment HEAO-3 C2 [14] (C2) and the Mass Spectrometer Telescope (MAST) on the Solar Anomalous and Magnetic Particle Explorer (SAMPEX) spacecraft [15]. The HEAO-3 satellite flew between 1979 and 1981 at an altitude of $496 \mathrm{~km}$ and inclination of $43.6^{\circ}$. From the comparison between the experimental and computed cutoffs of oxygen nuclei in the $5 \mathrm{GeV} / \mathrm{n}$ range it was found that the calculated cutoffs were systematically $\sim 3-5 \pm 2 \%$ higher than the measured ones [14]. The SAMPEX mission was launched into an orbit with $82^{\circ}$ inclination with an average altitude of $\sim 600 \mathrm{~km}$ and operated from 1992 to mid 2004. They performed a comparison between measured cutoff rigidities of $0.3-1.7 \mathrm{GeV}$ protons during geomagnetically quiet times with those predicted by the Smart and Shea tracer code (based on IGRF-10) and found that the latter systematically overestimate the data by $8-14 \%$ [16].

Directly comparing these findings with the measurements presented here is difficult because in both the SAMPEX and HEAO-3 cases the measured cutoff rigidities are much lower than those appropriate for Fermi. Smart and Shea in [17] assert that the accuracy of the model improves in regions closer to the geomagnetic equator (i.e. for larger cutoff rigidities). According to their analysis, it is reasonable to assume that the bias in the tracer output used in this analysis is no greater than $\sim 3-5 \%$ for the Mcllwain $L$ regions spanned by the Fermi observatory. However, this is just an extrapolation based on the measurements performed by HEAO-3 and SAMPEX at lower rigidities and a definitive answer on the value of this bias at the Fermi rigidities remains unknown.

The uncertainty of the hadronic component of the Monte Carlo simulations used to evaluate the residual contamination is estimated to be no greater than $20 \%$. Its contribution to the overall systematic uncertainty in the final measurement of the cutoff is small 


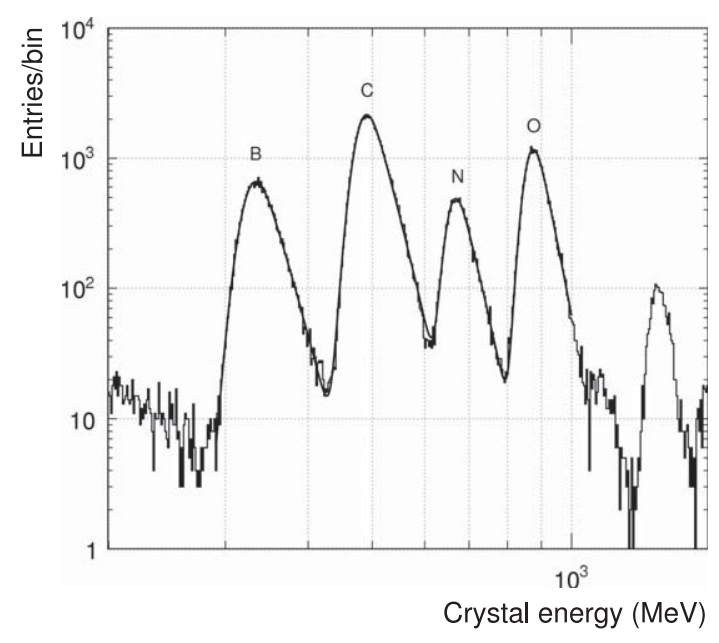

Fig. 6. The path length-corrected crystal energies of the $B, C, N$, and $O$ peaks corresponding to the first four months of Fermi LAT operation. The large apparent abundance of $\mathrm{B}$ (compared to secondary and primary Galactic cosmic-ray abundance) is due to secondary B produced by primary $C$ interacting in the ACD and TKR.

( $0.5 \%$ ) because the contamination is always below $20 \%$ in every McIlwain $L$ interval. The assessment of the systematic uncertainty due to the EGF is described in detail in [4] and for this energy range has a value of $10-15 \%$. We studied its effect on the final value of the cutoff via a bracketing method. To do this we generated a set of EGFs that have a maximal impact on the final shape of the spectrum, reconstructed the spectrum using these new EGFs and analyzed the final value of the cutoff energy obtained with these new EGFs. The resulting values varied by $\pm 1 \%$.

\subsection{Cross check of method}

To demonstrate that this analysis has the sensitivity to measure deviations of several percent in the energy scale of the LAT, we repeated the analysis on a test sample of traced particles with an added $+5 \%$ shift in energy. The resulting measurement of the cutoff energy was $5.4 \pm 0.2 \%$ higher, which is consistent with the bias we introduced in the input. Thus we conclude that indeed this method is capable of measuring energy scale errors of the magnitude we expect.

Pre-launch tests of crystal boule samples showed [18] that the light yield of the CSI ( Tl) crystals in the LAT calorimeter could be expected to decrease by $\sim 1 \%$ per year from radiation damage in the charged-particle environment of low Earth orbit, primarily from trapped particles in the South Atlantic Anomaly.

By analyzing the path length-corrected ${ }^{6}$ crystal energies of four abundant GCR elements, namely boron, carbon, nitrogen and oxygen, over time it is possible to verify this prediction with flight data. In fact, all four of these peaks show a similar linear decrease per year. These results are consistent with the pre-launch predictions. An example of these peaks is shown in Fig. 6. As a further cross check of the sensitivity of the method used in this analysis, we have compared the cutoff rigidity values measured in the first 60 days to the last 60 days of the first year of operations. We found that the measured cutoff energy decreased by $1.9 \pm 0.9 \%$ over this time interval, which is consistent with the decrease from radiation damage measured with the GCR element peaks, as can be seen in Table 1. We note also that this provides further confirmation of the sensitivity of our method.

\footnotetext{
${ }^{6}$ The energy deposit is normalized by $\cos \theta$, where $\theta$ is the incidence angle of the particle.
}

Table 1

Drift per year for four abundant GCR element (B, C,N, and O) peaks and the CRE cutoff energy measured in Fermi LAT flight data over the first year of operations.

\begin{tabular}{ll}
\hline & Drift $(\% / \mathrm{yr})$ \\
\hline $\mathrm{B}$ & $1.49 \pm 0.05$ \\
$\mathrm{C}$ & $1.60 \pm 0.04$ \\
$\mathrm{~N}$ & $1.50 \pm 0.06$ \\
$\mathrm{O}$ & $1.46 \pm 0.03$ \\
$\mathrm{CRE}$ & $1.90 \pm 0.90$ \\
\hline
\end{tabular}

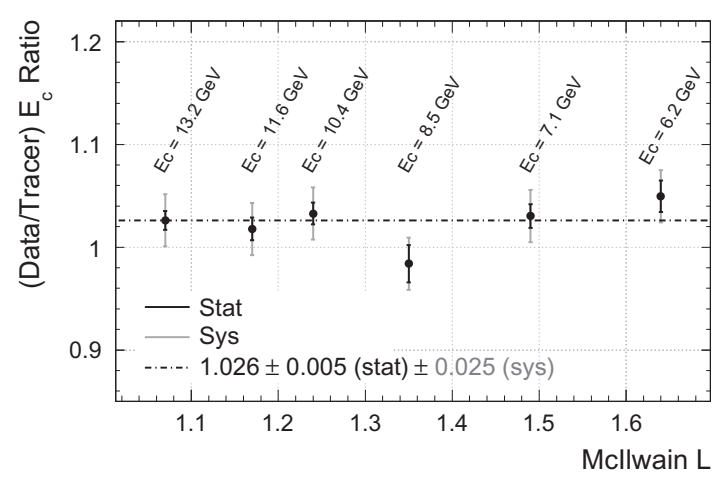

Fig. 7. Distribution of cutoff energy ratios as a function of Mcllwain $L$. The ratios are constant within the errors over the entire range. The quoted value for the ratio is the weighted mean. The statistical errors are represented by the black error bars, and the systematic errors are in gray. As a reference, the value for the cutoff energy in each Mcllwain $L$ interval is also shown.

\section{Results}

We applied this analysis to a data set spanning the first year of LAT operations. We found that the measured cutoff energy exceeded the predicted cutoff energy by $1.026 \pm 0.005$ (stat) \pm 0.025 (sys) in the $6 \mathrm{GeV}$ to $13 \mathrm{GeV}$ range. The systematic error is the sum in quadrature of our estimates in Section 4.3, excluding those from the uncertainty in the magnetic field model and particle tracing code. As shown in Fig. 7, the ratio between measured cutoff and predicted cutoff is constant within the errors over this energy range.

For the energy range in question-where the instrument acceptance is relatively flat-an energy-independent bias in the absolute energy scale translates into a rigid shift of the spectrum, scaling the normalization but leaving the spectral index unaffected. For the physically motivated case of a source with a power-law spectrum with index $\Gamma=-2$, the shift in the normalization is of the same amount as the bias. Therefore the effect of an error at the $\sim 2 \%$ level in the energy scale is smaller than other instrument-related sources of systematics (most notably the uncertainties in the effective area).

\section{Conclusions}

We provide a method for calibrating the LAT energy scale based on the measurement of the geomagnetic cutoff in the primary CRE spectrum. The results shown here confirm that the LAT calorimeter calibration procedure, based on measured electronic gains and non-linearities, observed proton signals, and enforced consistency between readout energy ranges, is accurate to the $\sim 2 \%$ level. It is worth noting that the method developed here is the only such calibration which relies uniquely on flight data and uses electromagnetic showers in the LAT. Accordingly, this is a very important cross check on other methods such as beam tests and calibrations with cosmic-ray heavy ions.

Our conclusion relies, however, on the fidelity of the current IGRF and particle trajectory tracing code. If we take the measurements of 
HEAO- 3 C 2 and SAMPEX to indicate a bias of the order of $+3-5 \%$, the absolute energy scale of the LAT may have to be decreased by $5-7 \%$. This conclusion, if confirmed, would make the energy scale more consistent with the beam test results and the Monte Carlo simulations of the CU. This would suggest that the on-orbit energy calibration technique for the LAT should be revised. However, the cross checks reported in $[14,16]$ on the predictions of the tracer code were both performed where the cutoff rigidities are much lower than those measured by the LAT and in geomagnetic regions where the trajectory tracing calculations are less accurate [7]. It is therefore unclear whether this bias in the theoretical cutoffs also applies to the results presented in this work. We therefore prefer to determine the energy scale of the LAT using the results of the proton inter-range calibration, which are confirmed by the independent measurements discussed in this paper.

\section{Acknowledgments}

The Fermi LAT Collaboration acknowledges generous ongoing support from a number of agencies and institutes that have supported both the development and the operation of the LAT as well as scientific data analysis. These include the National Aeronautics and Space Administration and the Department of Energy in the USA, the Commissariat à l'Energie Atomique and the Centre National de la Recherche Scientifique/ Institut National de Physique Nucléaire et de Physique des Particules in France, the Agenzia Spaziale Italiana and the Istituto Nazionale di Fisica Nucleare in Italy, the Ministry of Education, Culture, Sports, Science and Technology (MEXT), High Energy Accelerator Research Organization (KEK) and Japan Aerospace Exploration Agency (JAXA) in Japan, and the K.A. Wallenberg Foundation, the Swedish Research Council and the Swedish National Space Board in Sweden.

Additional support for science analysis during the operations phase is gratefully acknowledged from the Istituto Nazionale di Astrofisica in Italy and the Centre National d'Études Spatiales in France.

\section{References}

[1] W.B. Atwood et al., The large area telescope on the Fermi gamma-ray space telescope mission, ApJ 697 (2009) 1071-1102.

[2] A.A. Abdo et al., The first Fermi large area telescope catalog of gamma-ray pulsars, The Astrophysical Journal Supplement Series 187 (2010) 460-494.

[3] A.A. Abdo et al., The vela pulsar: results from the first year of Fermi LAT observations, ApJ 713 (2010) 154-165.

[4] M. Ackermann et al., Fermi LAT observations of cosmic-ray electrons from $7 \mathrm{GeV}$ to $1 \mathrm{TeV}$, Physical Reviews D 82 (2010) 092004

[5] A.A. Abdo et al., Measurement of the cosmic ray $e^{+}+e^{-}$Spectrum from $20 \mathrm{GeV}$ to $1 \mathrm{TeV}$ with the Fermi large area telescope, Physical Review Letters 102 (2009) 181101.

[6] M. Walt, Introduction to Geomagnetically Trapped Radiation, Cambridge University Press, 1994.

[7] D. Smart, M. Shea, A review of geomagnetic cutoff rigidities for earth-orbiting spacecraft, Advances in Space Research 36 (2005) 2012-2020. Solar windmagnetosphere-ionosphere dynamics and radiation models.

[8] A.A. Abdo et al., The on-orbit calibration of the Fermi large area telescope, Astroparticle Physics 32 (2009) 193-219.

[9] B. Lott et al., Response of the GLAST LAT calorimeter to relativistic heavy ions, Nuclear Instruments and Methods A560 (2006) 395-404.

[10] K. Nakamura, P.D. Group, Review of particle physics, Journal of Physics G: Nuclear and Particle Physics 37 (2010) 075021.

[11] L. Baldini, et al., Preliminary results of the LAT calibration unit beam tests, in: S. Ritz, P. Michelson, C.A. Meegan (Ed.), The First GLAST Symposium, of American Institute of Physics Conference Series, vol. 921, pp. 190-204.

[12] C.C. Finlay et al., International Geomagnetic Reference Field: the eleventh generation, Geophysical Journal International of Geomagnetism, 183 (2010) $1216-1230$

[13] O. Adriani et al., An anomalous positron abundance in cosmic rays with energies 1.5-100 GeV, Nature 458 (2009) 607-609.

[14] M. Bouffard, J.J. Engelmann, L. Koch, A. Soutoul, N. Lund, B. Peters, I.L. Rasmussen, The HEAO-3 cosmic ray isotope spectrometer, Astrophysics and Space Science 84 (1982) 3-33. 10.1007/BF00713625.

[15] D.N. Baker, G.M. Mason, O. Figueroa, G. Colon, J. Watzin, R.M. Aleman, An Overview of the solar, anomalous, and magneticspheric particle explorer (SAMPEX) mission, IEEE Transactions on Geoscience and Remote Sensing 31 (1993).

[16] R.C. Ogliore, R.A. Mewaldt, R.A. Leske, E.C. Stone, T.T. von Rosenvinge, A direct measurement of the geomagnetic cutoff for cosmic rays at space station latitudes, in: International Cosmic Ray Conference, volume 10, pp. 4112.

[17] D.F. Smart, M.A. Shea, Geomagnetic cutoffs: a review for space dosimetry applications, Advances in Space Research 14 (1994) 787-797.

[18] S. Bergenius Gavler et al., Radiation tests of CsI(Tl) crystals for the GLAST satellite mission, Nuclear Instruments and Methods A545 (2005) 842-851. 